\title{
Targeted delivery of mannosylated-PLGA nanoparticles of antiretroviral drug to brain
}

This article was published in the following Dove Press journal:

International Journal of Nanomedicine

\author{
Bhavin K Patel \\ Rajesh H Parikh \\ Nilesh Patel \\ Department of Pharmaceutics and \\ Pharmaceutical Technology, Ramanbhai \\ Patel College of Pharmacy, Charotar \\ University of Science and Technology, \\ Changa, Gujarat, India
}

\begin{abstract}
Mannosylated polymeric nanoparticles (NPs) enable improvement of brain bioavailability and reduction of dosing due to efficient drug delivery at the target site. Mannose receptors are present on the surface of macrophages, and therefore, in this study, it is expected that mannosylated NPs of anti-human immunodeficiency virus drug may target the macrophages, which may improve the therapeutic outcome and reduce the toxicity of antiretroviral bioactives. Poly(lactic-co-glycolic acid) (PLGA) and mannosylated-PLGA NPs (Mn-PLGA NPs) were prepared and administered by intravenous route in a dose of $10 \mathrm{mg} / \mathrm{kg}$. After predetermined time period, the pharmacokinetics and biodistribution of NPs were analyzed using high-performance liquid chromatography and confocal microscopy, respectively. Results of this study indicated that Mn-PLGA NPs would be a promising therapeutic system for efficient delivery of the drug into brain macrophages.
\end{abstract}

Keywords: mannosylated poly(lactic-co-glycolic acid) nanoparticles, brain targeting, macrophage targeting, human immunodeficiency virus

\section{Introduction}

Infectious diseases caused by intracellular virus represent a challenge for current antiviral therapies. Insufficient concentrations and very short residence time of the anti-HIV drugs at the cellular and infectious sites are among the major problems that contribute to the failure of eradicating HIV from reservoirs and the development of multidrug resistance against these anti-HIV drugs. Lamivudine is an antiretroviral drug belonging to the category of reverse transcriptase inhibitors. The viruses reside in the macrophages and they cannot be killed completely by conventional therapy due to poor intracellular targeting. The brain bioavailability of lamivudine is $0.05 \%-1.14 \%$, whereas the virologic $\mathrm{IC}_{50}$ of lamivudine in brain is $0.78 \%-4.90 \% .{ }^{1}$ Hence, it is required to have sufficient drug concentration in the brain macrophages. Mannosylated polymeric nanoparticles (NPs) enable improvement of brain bioavailability and reduction of the dosing due to efficient drug delivery at the target site. ${ }^{2,3}$ In addition, the NPs may internalize through the blood-brain barrier (BBB) and enter into the brain stream by enhanced permeation retention effect. Mannose receptors are present on the surface of macrophages, and therefore, it is expected that mannosylated NPs of anti-HIV drug may target the macrophages, which may improve the therapeutic outcome and reduce the toxicity of antiretroviral bioactives.

\section{Materials and methods Materials}

Poly(lactic-co-glycolic acid) (PLGA) was obtained as a gift sample from Evonik (Mumbai, India). Mannose was sourced from Sigma-Aldrich (Mumbai, India). 
Lamivudine was a gift from West Cost Pharmaceuticals Pvt. Ltd. (Ahmedabad, India) and was of pharmacopeial grade. All other chemicals were of analytical grade.

\section{Methods}

PLGA and mannosylated-PLGA NPs (Mn-PLGA NPs) were prepared by water in oil in water double-emulsion-solvent evaporation method with some modification. ${ }^{3,4}$ A $3^{2}$ full factorial design was used to study the effect of independent variables on dependent variables, as indicated in Table 1. Particle size and zeta potential of the formulation were measured using dynamic light scattering technique. Morphologic characteristics of the NPs were visualized using transmission electron microscopy. Lectin-binding assay was performed to ensure the conjugation of mannose to PLGA. ${ }^{5}$ In vitro drug release study of the polymeric NPs was performed using dialysis bag diffusion technique in phosphate-buffered saline ( $\mathrm{pH} 7.4$ ) and sodium acetate buffer ( $\mathrm{pH}$ 5.5) to simulate the physiologic $\mathrm{pH}$ and macrophage environment, respectively. The Committee for the Purpose of Control and Supervision of Experiments on Animals (CPCSEA) is a statutory committee of the Government of India that was established under the provisions of Prevention of Cruelty to Animals Act 1960. The Institutional Animal Ethics Committee (IAEC) of Ramanbhai Patel College of Pharmacy (RPCP) was constituted as per the norms laid down by the CPCSEA. The IAEC of RPCP granted approval for the use of animals (rats) as well as to conduct the experiment with the approved protocol number RPCP/ IAEC/2013-2014/MPH-PT-60. The guidelines issued by the CPCSEA were followed for the care and safety of animals used in the study. Drug solution, PLGA NPs and Mn-PLGA NPs were administered by intravenous (IV) route at a dose of $10 \mathrm{mg} / \mathrm{kg}$. At predetermined time intervals, blood samples were collected and brain was isolated and analyzed by highperformance liquid chromatography to study the in vivo pharmacokinetic behavior. Rhodamine dye-labeled Mn-PLGA NPs were administered by IV route and the biodistribution of the therapeutic systems into the brain was observed using a confocal microscope. In vitro activation of macrophages was induced by concanavalin A $(1 \mathrm{mg} / \mathrm{mL})$ and they were treated with FITC-labeled PLGA NPs and Mn-PLGA NPs. Cells were harvested and analyzed under a fluorescence microscope (DM 2500; Leica, Wetzlar, Germany).

\section{Results and discussion \\ Characterization of NPs}

Table 1 shows the particle size and the drug entrapment efficiency of the prepared NPs. The results were subjected to statistical evaluation using design expert software (Version 8.0; Stat Ease, Minneapolis, MN, USA) and the following polynomial equations were derived:

$$
\begin{aligned}
Y_{1}= & 614.46-10.34 X_{1}+54189 X_{2}+0.0758 X_{1}^{2} \\
& +2.3266 X_{2}^{2}+0.01044 X_{1} X_{2} \\
Y_{2}= & 134.05-0.90 X_{1}+0.02466 X_{2} \\
Y_{3}= & 90.75-0.47 X_{1}+0.11458 X_{2}
\end{aligned}
$$

It can be observed that the volume of the external phase of secondary emulsion $\left(\mathrm{X}_{1}\right)$ had a negative effect on the particle size, entrapment efficiency and drug loading. In contrast, the amount of polymer $\left(\mathrm{X}_{2}\right)$ had a positive effect on the particle size, entrapment efficiency and drug loading. The P4 batch was found to be optimized as per the desirability function.

\begin{tabular}{|c|c|c|c|c|c|c|}
\hline \multirow[t]{2}{*}{$\begin{array}{l}\text { Batch } \\
\text { code }\end{array}$} & \multicolumn{2}{|c|}{$\begin{array}{l}\text { Independent } \\
\text { variables }\end{array}$} & \multicolumn{3}{|c|}{ Dependent variables } & \multirow[t]{2}{*}{ OD } \\
\hline & $x_{1}$ & $x_{2}$ & $\begin{array}{l}\text { Average particle } \\
\text { size }(n=3)\left(Y_{1}\right)\end{array}$ & $\begin{array}{l}\% \text { Drug entrapment } \\
\text { efficiency }(n=3)\left(Y_{2}\right)\end{array}$ & Drug loading $\left(\mathbf{Y}_{3}\right)$ & \\
\hline PI & -1 & -1 & $188.9 \pm 3.6$ & $91.24 \pm 2.6$ & 40.61 & 0.7854 \\
\hline P2 & -1 & 0 & $198.1 \pm 2.38$ & $88.19 \pm 0.6$ & 39.81 & 0.7137 \\
\hline P3 & -1 & +1 & $272.4 \pm 2.98$ & $88.34 \pm 0.20$ & 30.64 & 0.197 \\
\hline $\mathbf{P 4}$ & 0 & -1 & $174.2 \pm 12.10$ & $84.3 \pm 1.42$ & 55.84 & 0.997 \\
\hline P5 & 0 & 0 & $192.5 \pm 0.92$ & $83.4 I \pm 0.87$ & 29.43 & 0.5206 \\
\hline P6 & 0 & +1 & $251.17 \pm 3.45$ & $81.95 \pm 0.57$ & 23.50 & 0.2415 \\
\hline P7 & +1 & -1 & $180.33 \pm 10.69$ & $77.50 \pm 0.89$ & 36.75 & 0.5597 \\
\hline P8 & +1 & 0 & $195.9 \pm 0.7$ & $67.75 \pm 3.93$ & 25.30 & 0 \\
\hline P9 & +1 & +1 & $275.6 \pm 3.4$ & $67.95 \pm 2.4$ & 20.31 & 0 \\
\hline
\end{tabular}

Zeta potential of the optimized batch (P4) was found to be $-46.913 \mathrm{mV}$. Mn-PLGA NPs had a negative charge

Table I Independent and dependent variables

Notes: $\mathrm{X}_{1}$, volume of the external phase of the secondary emulsion; $\mathrm{X}_{2}$, amount of the polymer. Bold data indicates nanoparticles with the highest overall desirability. Abbreviation: OD, overall desirability. 


\section{A In vitro drug release study}

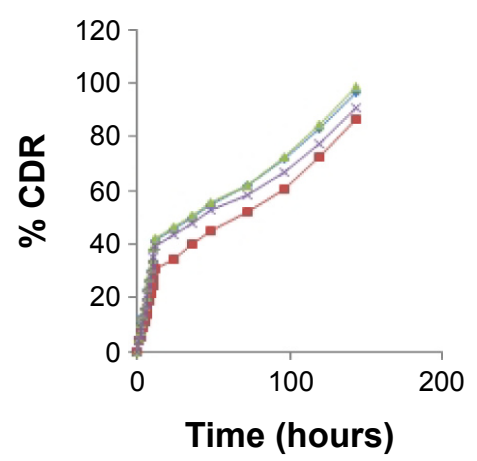

— PBS (M-PLGA) $\rightarrow$ PBS (PLGA)
$\approx$ Sodium acetate (M-PLGA)
$\approx$ Sodium acetate (PLGA)

\section{B Brain/plasma ratio (IV)}

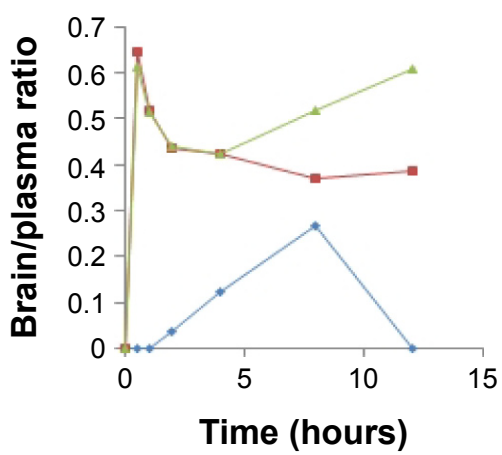

$\because$ Drug solution $\rightarrow$ PLGA NPs
$\because$ Mn-PLGA NPs

Figure I (A) In-vitro drug release study. (B) In-vivo pharmacokinetic study: brain/plasma ratio of the drug solution, lamivudine-loaded PLGA and Mn-PLGA NPs. Abbreviations: CDR, cumulative drug release; IV, intravenous; Mn-PLGA, mannosylated PLGA; NPs, nanoparticles; PBS, phosphate-buffered saline; PLGA, poly(lacticco-glycolic acid).

due to masking of the free carboxylic group by the negatively charged oligosaccharide utilized for conjugation. Transmission electron microscopic study showed that MnPLGA NPs were relatively less spherical when compared with PLGA NPs. In vitro drug release study showed sustained release from the prepared NPs in both release media up to 6 days, as shown in Figure 1A. It is clearly evident from lectin-binding assay that mannose has been successfully conjugated with PLGA, and hence, it has higher susceptibility to interact with the lectin receptors than PLGA NPs.

\section{In vivo pharmacokinetic study}

The calculated brain/plasma ratio following the IV administration of Mn-PLGA and PLGA NPS was found to be significantly higher at all time points, compared to the drug solution as shown in Figure 1B. The concentration of lamivudine was continuously increased up to 12 hours in MnPLGA NPs. It proves the effective targeted delivery to the brain through the $\mathrm{BBB}$, which may be due to the presence of sugar receptors on the luminal surface of $\mathrm{BBB}$.

\section{Biodistribution study}

Results of confocal imaging study showed that the internalization of rhodamine-labeled Mn-PLGA NPs across the BBB was done successfully.

\section{Cellular uptake study}

Figure 2 shows that there was higher fluorescence intensity of Mn-PLGA NPs compared to PLGA NPs in the macrophages, suggesting that Mn-PLGA NPs had higher susceptibility for macrophage uptake compared to the PLGA NPs because of the specific interaction between the mannose molecules with the mannosyl receptor.

\section{Conclusion}

Surface-functionalized Mn-PLGA NPs of lamivudine developed as part of the present research work would be a promising therapeutic system for efficient delivery of the drug into brain macrophages. Therefore, it seems to have potential as an immunotherapeutic agent for the treatment of HIV-1.
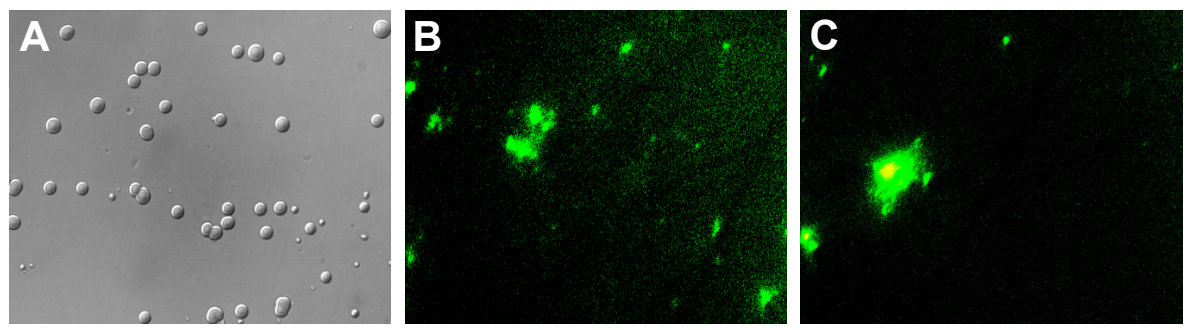

Figure 2 (A) Blank macrophages; (B) FITC-labeled Mn-PLGA NPs; (C) FITC-labeled PLGA NPs. Magnification 500x. Abbreviations: Mn-PLGA, mannosylated PLGA; NPs, nanoparticles; PLGA, poly(lactic-co-glycolic acid). 


\section{Acknowledgment}

The facility and funding for this study were provided by Charotar University of Science and Technology (CHARUSAT), Gujarat, India.

\section{Disclosure}

The authors report no conflicts of interest in this work.

\section{References}

1. Sergio MA, Scott L, Ron E. The human immunodeficiency virus and the central nervous system. Braz J Infect Dis. 2006;10(1):41-50.

2. Fansheng K, Fang Z, Linfu G, Ximin L, Yong W. Mannosylated liposomes for targeted gene delivery. Int J Nanomedicine. 2012;7:1079-1089.
3. Saraogi GK, Jain NK. Mannosylated gelatin nanoparticles bearing isoniazid for effective management of tuberculosis. J Drug Target. 2011; 19(3):219-227.

4. Parikh RH, Parikh JR, Dubey RR, Soni HN. Poly (D, L-lactide-coglycolide) microspheres containing 5-fluorouracil: optimization of process parameters. AAPS Pharm SciTech. 2003;4(2):14-21.

5. PalanirajanVK, Asthana A, Dutta T, Jain NK. Intracellular macrophage uptake of rifampicin loaded mannosylated dendrimers. J Drug Target. 2006;14(8):546-556.

\section{Publish your work in this journal}

The International Journal of Nanomedicine is an international, peerreviewed journal focusing on the application of nanotechnology in diagnostics, therapeutics, and drug delivery systems throughout the biomedical field. This journal is indexed on PubMed Central, MedLine, CAS, SciSearch $\AA$, Current Contents ${ }^{\circledR} /$ Clinical Medicine,
Journal Citation Reports/Science Edition, EMBase, Scopus and the Elsevier Bibliographic databases. The manuscript management system is completely online and includes a very quick and fair peer-review system, which is all easy to use. Visit http://www.dovepress.com/ testimonials.php to read real quotes from published authors. 